\title{
Characteristic of Acoustic Signals Generated by Operation of On Load Tap Changers
}

\author{
A. Cichon*, P. Fracz And D. Zmarzly \\ Faculty of Electrical Engineering, Automatic Control and Computer Science, Opole University of Technology \\ S. Mikołajczyka 5, 45-271 Opole, Poland
}

Possibilities of application of the acoustic method for the on load tab changers diagnosis has been presented in the paper. The analysis of measurement results gathered during research works performed under laboratory conditions has been presented in detail. The registered acoustic signals have been analyzed in time and time-frequency domains. The time domain analysis has been performed by presentation of time runs and their envelopes determined by use of the Hilbert transform. Evaluation of the time-frequency components has been performed by use of the short-time Fourier transform, the discrete and the continuous wavelet transforms.

PACS: 52.80.-s, 43.28.Js

\section{Introduction}

Power transformers are a significant part of assets owned by entities distributing and transferring the electric energy. In Poland there are several thousands of medium and high power transformers in operation, while over $50 \%$ of them are older than 30 years which means that they have already exceeded their planned lifetime. The main elements of transmission grids are power transformers mostly equipped with on load tab changers (OLTCs). The most common operational problems connected with OLTC may be presented as follows: wear or damage of the main contacts, wear of the resistance contacts, break or improper pull of the power springs, defects of the power transmission system (clearance on transmissions, the Cardan joints etc.) [1-6].

In spite of a relatively long operation time of transformers in service in the Polish electric power system, the technical condition of most of them is good enough to be used for the next few years. A growing demand for the electric energy, maintenance works and limited possibilities of switch-overs resulting from the network structure cause that in practice problems connected with necessary shut-downs of power transformers for periodical diagnostic tests are encountered more and more often. This issue appertains mainly to high-power transmission transformers and less to distributive transformers supplying strategic industrial consumers. Therefore, it becomes necessary to look for new diagnostic methods for the on-line assessment of the OLTC operation condition, e.g. without shutting-down the transformer under study $[1,3,7]$.

\footnotetext{
* corresponding author; e-mail: a.cichon@po.opole.pl
}

A method which makes online diagnosis of devices possible is the acoustic method; it is applied for technical condition evaluation of power transformer windings insulation with regard to partial discharges appearance. The paper presents preliminary results of investigations considering description of the acoustic signals generated during changer operation and application possibility of this method for the OLTC technical condition estimation.

\section{The measurement setup}

As already mentioned all measurements are performed under laboratory conditions. The main element of the setup is a tank, dimensions: $50 \times 60 \times 80 \mathrm{~cm}^{3}-$ especially designed for the measurement purposes, which enables for location of a tab changer in the middle of it; the tab changer is fixed at the bottom of the tank. In order to bring closer the laboratory conditions to conditions present during operation of tab changers in power transformers the tank has been filled with insulation oil. Figure 1a depicts the OLTC type VEL-110, made by ELIN applied in the measurements. For separation from outer acoustic signals the measurements have been performed in a measurement cage, where the walls have been covered with a special acoustic insulation. Figure 1b depicts general view of the measurement cage.

The acoustic signal has been measured by use of a wideband measurement transducer type WD AH 17, made by PAC, which was mounted on the outer surface of the tank with a magnetic chuck. The transducer applied in measurements is of high sensibility $(55 \mathrm{~dB} \pm 1.5 \mathrm{~dB}$ with respect to $\mathrm{V} / \mathrm{ms}^{-1}$ ) and of wide frequency band from $100 \mathrm{kHz}$ to $1 \mathrm{MHz}$ in the range of $\pm 10 \mathrm{~dB}$. The acoustic signal measured by the transducer has been amplified in a preamplifier configuration type $2 / 4 / 6$, made 


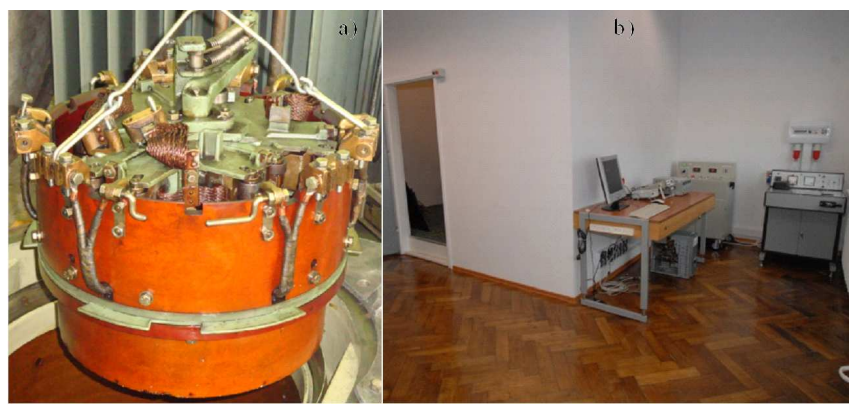

Fig. 1. The changer type VEL-110 (a), the measurement cage with acoustic insulation (b).

by PAC. Further the acoustic signal is forwarded to an amplifier configuration where it is amplified by value of $20 \mathrm{~dB}$. Moreover, the setup includes a band pass filter with cut-off frequencies 10 and $500 \mathrm{kHz}$. The measurement setup is presented in Fig. 2.

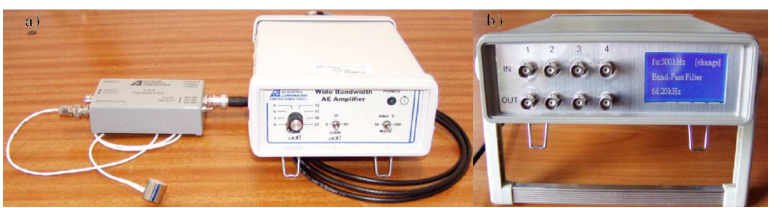

Fig. 2. Measurement setup: (a) transducer WD AH 17 with preamplifier and amplifier (b) band pass filter.

\section{Analysis of measurement results}

Figure 3 depicts time run of the acoustic signals generated during operation of the OLTC.

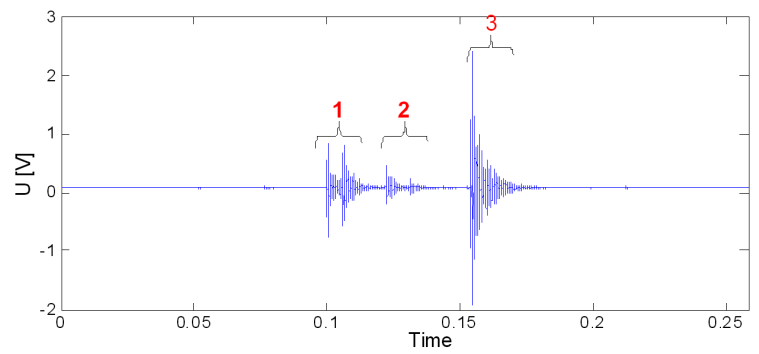

Fig. 3. Time run of the acoustic signal generated by OLTC.

The acoustic signal registered during operation of the OLTC contains time structures which mark out due to their characteristic shape and are specific for particular device. In the case of changer type VEL-110, applied in the laboratory and industrial investigations, in the acoustic signal one can distinguish three structures that are marked by numbers one, two and three in Fig. 4. The designation system adopted for the successive acoustic events appearing in the investigated signal will be later applied during results evaluation presented in the subsequent part of this paper. The structure no. three is characterized by its single maximum, while in structures one and two, two components with different amplitudes can be distinguished. In order to expose the time structures the analyzed signal has been processed by use of the Hilbert transform where an envelope of the measured signal was determined for a shortened time interval (see Fig. 4).

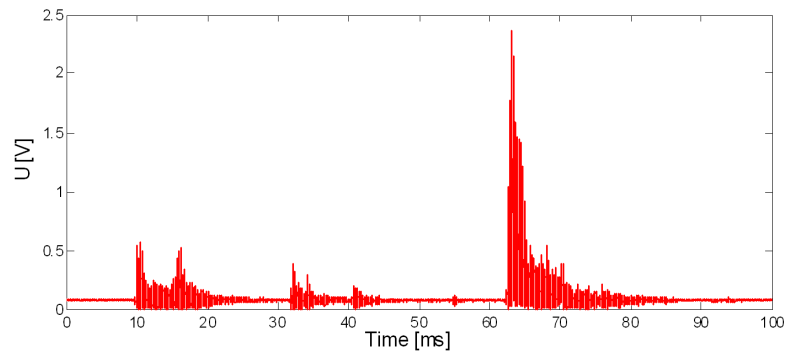

Fig. 4. Envelope of the acoustic signals generated by OLTC determined for a shortened time interval.

For measurable description of the time intervals between the particular structures the following characteristic times have been determined between structures (see Table I):

$$
\begin{aligned}
& \text { — first and second - T1; } \\
& \text { — second and third - T2; } \\
& \text { — first and third - Tc. }
\end{aligned}
$$

TABLE I

The characteristic times determined for the acoustic signals generated by OLTC.

\begin{tabular}{c|c|c}
\hline \hline $\mathrm{T} 1[\mathrm{~ms}]$ & $\mathrm{T} 2[\mathrm{~ms}]$ & $\mathrm{Tc}[\mathrm{ms}]$ \\
\hline 22.0 & 30.6 & 52.6
\end{tabular}

The time-frequency analysis has been performed by use of the following conversions: short time Fourier transform (STFT), continuous wavelet transforms (CWT), discrete wavelet transforms (DWT). Results of the STFT are shown in Fig. 5 in form of two-dimensional power spectral density diagram.

The time-frequency components observed in the spectrum arise in the time interval between 100 and $200 \mathrm{~ms}$; frequency components appear in the range from 10 to $500 \mathrm{kHz}$. Due to the amplitude value and duration time as notably significant the range from 10 to $50 \mathrm{~Hz}$ can be conceded. Components with increased amplitude are to be recognized in the range from 100 to $120 \mathrm{kHz}$. The time-frequency patterns above $120 \mathrm{kHz}$ are characterized by short duration time and also by smaller amplitude. For additional accuracy the time-frequency analysis performed by use of the STFT was extended by the CWT 


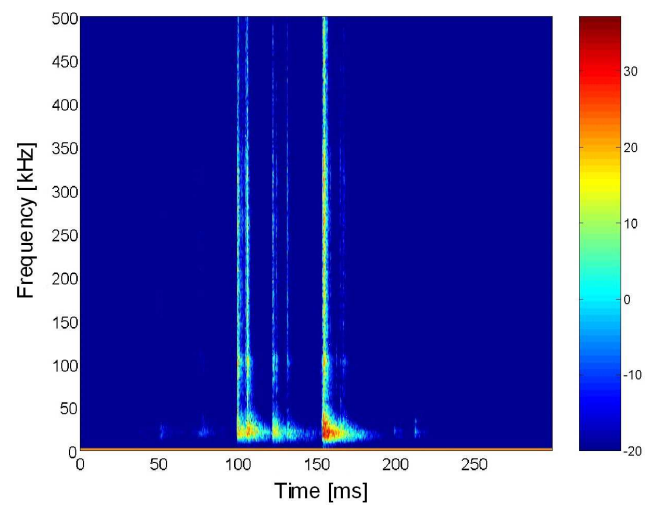

Fig. 5. Power spectrum density of the acoustic signals generated by OLTC.

conversion. The CWT diagram, see Fig. 6, was determined for a time interval shortened to $120 \mathrm{~ms}$.

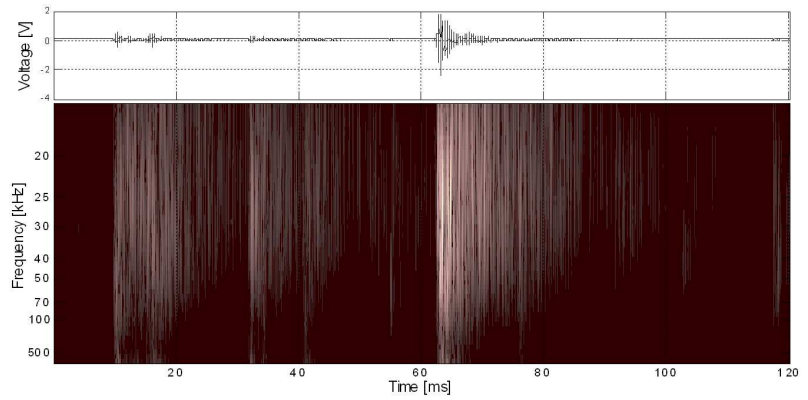

Fig. 6. The CWT diagram of the acoustic signals generated by OLTC.

In the CWT diagram, which is shown in Fig. 6, three time-frequency structures regarding to the successive changer switch-over phases was presented. The wave structures corresponding to the first acoustic event are characterized by frequency content in range from 0 to $100 \mathrm{kHz}$. Components above this frequency have small amplitude and are characterized by short duration time. The frequency band in the range from 0 to $70 \mathrm{~Hz}$ is characteristic for the second acoustic event. The highest amplitude and the longest duration time appear in fluctuations in the third structure. Except for dominant participation of low frequency band this structure is characterized by components of large amplitude and short fluctuations duration time in frequency range above $100 \mathrm{kHz}$.

Extension of the wavelets analysis performed by use of the CWT is the DWT. The frequency ranges regarding to filter bands for the analyzed details are presented in Table II.

In the context of graphical results presentation for the acoustic signals generated by power changer the following parameters have been determined: time runs, seventh decomposition level approximation and runs of seven details. In order to determine magnitude of the energy delivered by the particular details additionally the col-
TABLE II

The frequency bands applied for the particular details.

\begin{tabular}{c|c|c}
\hline \hline Details & $\begin{array}{c}\text { Middle frequency } \\
{[\mathrm{kHz}]}\end{array}$ & $\begin{array}{c}\text { Bandwidth } \\
{[\mathrm{kHz}]}\end{array}$ \\
\hline D1 & 375 & $250-500$ \\
D2 & 187.5 & $125-250$ \\
D3 & 93.75 & $62.5-125$ \\
D4 & 46.87 & $31.25-62.5$ \\
D5 & 23.43 & $15.62-31.25$ \\
D6 & 11.71 & $7.81-15.62$ \\
D7 & 5.86 & $3.91-7.81$
\end{tabular}

umn diagrams have been estimated. Analysis results of the DWT are presented in Fig. 7.

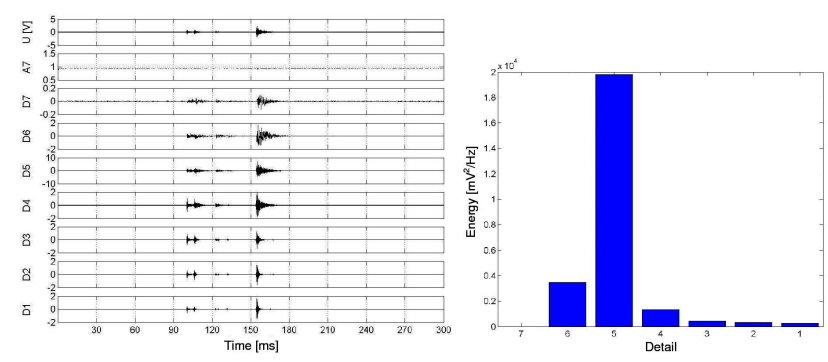

Fig. 7. Discrete wavelet decomposition and magnitude of the energy delivered by the particular details performed for the acoustic signals generated by OLTC.

In all runs corresponding to the particular decomposition levels three characteristic time structures occur. Fluctuations appearing in decomposition levels: 4, 5, 6 and 7 are characterized by the longest duration time. Events with the highest amplitude occur in detail D5 while an event with the smallest amplitude is characterized by detail D7. Large dynamics and significant amplitude are marked out by details D4 and D6. Comparing the wavelet decomposition runs and the column diagrams it can be stated that in the investigated signal frequencies dominate corresponding to details D4, D5 and D6. Details D1, D2, D3 and D7 do not significantly influence results of the time-frequency analysis of the investigated acoustic signals generated by the power changer. The frequency content in the range from 7.8 to $62.5 \mathrm{kHz}$ is in majority consistent with results achieved by application of the STFT and CWT.

\section{Summing-up}

In the analyzed acoustic signal, which was registered during operation of the OLTC, one can recognize structures corresponding to the consecutive switch-over cycles. Characteristics of the signal can be applied as diagnosis 
descriptor in evaluation of the OLTC technical condition. Description of acoustic signals generated by OLTC was presented in the time and in the time-frequency domains and applied for result interpretations. The results analysis should be based on comparison of the achieved results and results constituting a data base, a so-called "finger print" determined for the particular OLTC type. Results of scientifically investigative works performed imply theoretically and practically possibilities of the acoustic method application for the OLTC diagnosis. Within the scope of future research works measurements in configurations with modeled OLTC damages and with real objects are planned.

\section{Acknowledgments}

The research work is co-financed by the National Centre for Research and Development.

\section{References}

[1] A. Cichon, Acta Phys. Pol. A 116, 290 (2009).

[2] J.J. Erbrink, E. Gulski, P.P. Seitz, R. Leich, Int. Symp. Electrical Insulation ISEI, IEEE, Vancouver 2008, p. 252.

[3] M. Foata, R. Beauchemin, C. Rajotte, in: Proc. IEEE ESMO, Paris 2000, p. 293.

[4] E. Rivas, J.C. Burgos, J.C. García-Prada, IEEE Trans. Power Delivery 24, 687 (2009).

[5] E.F. Simas, L.A. Filho, L. de Almeida, in: IEEE Int. Instrumentation and Measurement Technology Conf., Victoria, 2008, p. 17.

[6] R. Vilaithong, S. Tenbohlen, T. Stirl, On-Line Tap Changer Diagnosis Based on Acoustic Technique, MatPost, Glasgow 2007.

[7] Transformers in Operation, Ed. J. Subocz, Energo-Complex, Opole 2007 (in Polish). 